\title{
An Efficient Continuous Flow Technique for Investigating the Magnetic Field Dependence of Photochemical Quantum Yields
}

\author{
Wolfgang Schlenker \\ Universităt Stuttgart, Pfaffenwaldring 55, 7000 Stuttgart 80, Federal Republic of Germany \\ Ulrich E. Steiner*) \\ Universität Konstanz, 7750 Konstanz, Federal Republic of Germany
}

Magnetic Field Effects / Methods and Systems / Photochemistry

A continuous-flow technique has been applied to study the magnetic field effect on the photobleaching quantum yield of thionine in the reaction with aniline in reversed micellar solution and with p-iodoaniline in methanol. The principle of the experimental technique is to irradiate the solution in a flow-through cuvette within a magnetic field which is varied in steps and to observe optical density changes in a second cuvette connected to the first one in a flow system. Although the absolute quantum yields of the two photoreactions are below $1 \%$, relative changes due to the magnetic field effect could be resolved with an accuracy of $1 \%$. The observed effects on the permanent photobleaching yield closely follow the effects on the radical yield previously observed in time-resolved experiments and are explained by the radical pair mechanism and the triplet mechanism, respectively.

\section{Introduction}

Many photochemical reactions involve paramagnetic intermediates, e.g. excited triplet states or radicals, the reactive

*) To whom correspondence should be addressed. behaviour of which may be influenced in a characteristic way by means of an external magnetic field [1 -3$]$.

Although the diagnostic value of such magnetic field effects for mechanistic purposes is comparable to that of the photo-CIDNP technique [4-6] their application is not as 
common as of the latter. The reason for this should be sought in the fact that CIDNP effects generally cause quite drastic changes of NMR spectra, whereas magnetic field effects on photochemical quantum yields are usually small and often escape observation due to the difficulties to measure relative quantum yields with sufficient accuracy.

Photochemical quantum yields are usually obtained from continuous illumination experiments. Considering e.g. a photoreaction of the general type $\mathrm{A}+h v \rightarrow \mathrm{B}$ the corresponding rate law is of the form

$\frac{\mathrm{d} c_{\mathrm{A}}}{\mathrm{d} t}=-\phi_{\mathrm{AB}} \cdot \Gamma \cdot f\left(c_{\mathrm{A}} \varepsilon_{\mathrm{A}}, c_{\mathrm{B}} \varepsilon_{\mathrm{B}}\right)$

which involves the quantum yield $\phi_{\mathrm{AB}}$, the actinic light flux $I$ and a so-called photokinetic factor $f$, expressing the fraction of light absorbed by the reacting species A. Rate laws of this type may be integrated in closed form only in simple cases and are generally treated by numerical methods [7].

Evaluating the differential form of the rate law, i.e. using the slope of $c(t)$ curves at the beginning of the reaction, is a simpler and quicker method but generally of much less accuracy, since the chemical conversion has to be kept low.

The reproducibility of photokinetic measurements can be greatly improved if irradiation and spectrophotometric detection of the reaction progress are performed in one instrument, preferably in the cuvette housing of a commercial UV/VIS absorption spectrometer. With such a set up, however, it is difficult at the same time to apply a magnetic field, especially if field strengths up to 1 Tesla and more are required. If, on the other hand, photochemical reactions in a magnetic field are performed and analyzed by repeatedly transferring one cuvette between separate positions for irradiation and spectrophotometry, the accuracy of the spectroscopic analysis suffers and. without averaging many repeated experiments, it may be difficult to satisfactorily assess magnetic field effects, especially if they are small.

In this paper we describe an efficient, though simple, experimental method allowing for quick, accurate and easyto-evaluate spectroscopic measurements of magnetic field effects on photochemical quantum yields.

Our method is comparable to the transfer method [8] used in CIDNP investigations, whereby samples are irradiated in variable magnetic fields outside the NMR cavity and are transferred to the latter by use of a continuous flow system. In our technique we use two optical flow-through cuvettes connected in series in a flow system, whereby the first cuvette is exposed to the actinic light in the field of an electromagnet. The solution to be investigated passes through this cuvette, where for a few seconds it is irradiated and then transferred in continuous flow to a second cuvette positioned in the analyzing light beam of a UV/VIS spectrophotometer. This instrument is set to follow the optical density at a fixed wavelength and the signal is recorded with great amplification using a suitably large offset. Sudden changes of the quantum yield, which may be induced by changing an external magnetic field, are reflected as marked steps of the signal trace. Under suitable conditions these steps are proportional to the change of the quantum yield. Thus, the magnetic field influence on the photochemical quantum yield can be directly seen from the height of the steps which are imposed on the signal traces as the magnetic field is changed in a stepwise fashion.

\section{Experimental}

\section{Apparatus}

The experimental set-up used for measuring magnetic-field dependent photochemical quantum yields is schematically shown in Fig. 1. The illumination part of the apparatus is as normally used in continuous illumination experiments, comprising a $500 \mathrm{~W}$ high pressure mercury lamp combined with heat filter, interference filter and neutral density filters. Using a quartz plate beam splitter, a constant fraction of the actinic light is separated for recording its intensity by means of a photo-diode. The actinic light is focussed onto the $4 \times 11 \mathrm{~mm}$ aperture of a flow-through cuvette (Hellma 164 $00-\mathrm{QS}$ ) which is positioned in the homogeneous field of an electromagnet (Drusch EAF $8 \mathrm{AU}$ ) the power supply of which allows for adjusting the magnetic field between $10^{-4}$ and 1 Tesla. The actual value of the magnetic field was recorded on a gaussmeter (Bell 615) by means of a Hall probe (Bell FTG 1-0415).

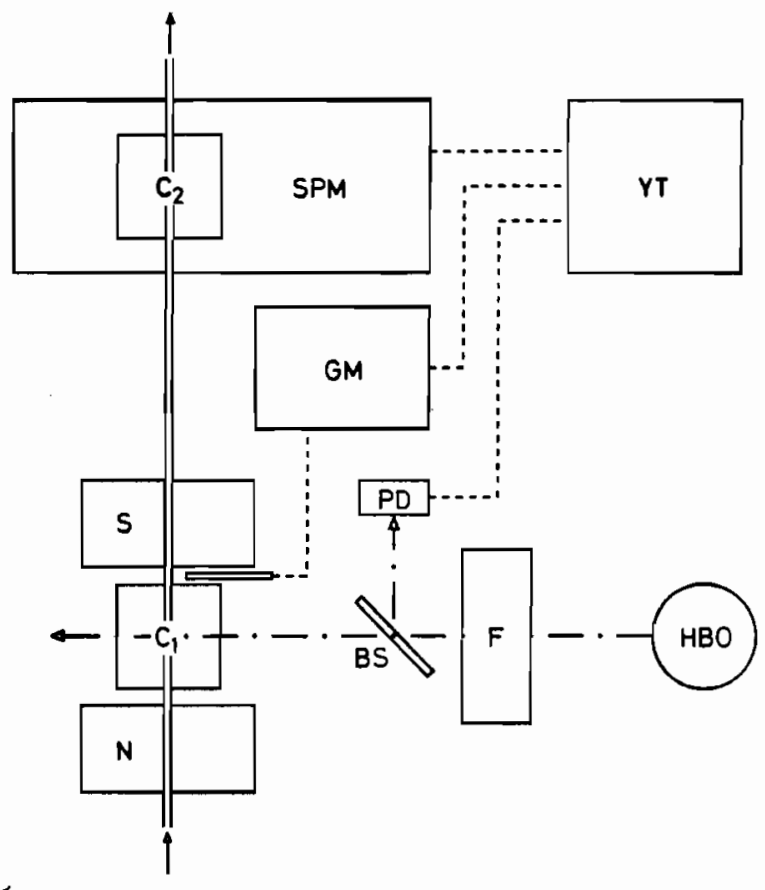

Fig. 1

Schematic diagram of the continuous flow apparatus for observing magnetic field effects: $B S$ beam splitter, $C_{1}, C_{2}$ flow-through cuvettes, $F$ filter packet, $G M$ gaussmeter with Hall probe, $\mathrm{HBO}$ mercury high pressure lamp, N, S electromagnet, PD photo diode, SPM spectrophotometer, YT three-channel pen recorder

The illumination cuvette is part of a flow-through system, wherein the flow of the reaction solution is driven by a $200 \mathrm{mbar}$ transport pressure of nitrogen in the support vessel. Using a needle valve, the flow is regulated to a typical flow rate of about $7.5 \mathrm{ml} /$ min, which corresponds to a mean residence time of $3.5 \mathrm{~s}$ for the solution in the illumination chamber of the flow-through cuvette. For the flow line a teflon pipe of $1.5 \mathrm{~mm}$ cross section is used which is contained in a $5 \mathrm{~mm}$ wide black silicone pipe flushed with nitrogen in order to prevent uncontrolled illumination and oxygen penetration from the ambient atmosphere. From the illumination cuvette the solution passes to a second flow-through cuvette of the same kind which is positioned in a spectrophotometer (Zeiss PMQ 3, single beam) where the actual optical density of the solution is recorded at a fixed wavelength. The signals of spectrophotometer, gaussmeter and photo-diode are simultaneously recorded on a three-channel Y/T plotter (Servogor 460-03). 


\section{Materials}

Thionine (Merck) was purified as described in Ref. [9]. Aniline (from Merck, p.A.) was distilled under vacuum, p-iodoaniline (Merck-Schuchardt) was recrystallized several times from n-hexane solutions after stirring them for some time with activated carbon, and was then sublimated under vacuum. Cetyldimethylbenzylammoniumchloride, henceforth denoted as CDBA, was obtained from Fluka (purum) and recrystallized three times from ethylacetate with $20 \%$ of n-hexane after treating the solutions with activated carbon. Solvents used were benzene (Merck, zur Rückstandsanalyse), methanol (Merck, p.A.) and deionized, doubly distilled water.

For the photochemical runs the following concentrations were used

I) (reversed micellar solution) $0.04 \mathrm{M} \mathrm{CDBA}, 0.54 \mathrm{M}$ water, $6 \cdot 10^{-6} \mathrm{M}$ thionine, $5 \cdot 10^{-4} \mathrm{M}$ aniline in benzene as bulk solvent.

II) (methanolic solution) $10^{-5} \mathrm{M}$ thionine, $10^{-3} \mathrm{M}$ p-iodoaniline.

Before the flow runs were started the solutions and the whole flow system were flushed with nitrogen (with oxygen content less than $6 \mathrm{ppm}$ ) for 45 minutes.

\section{Results}

\subsection{Relative Photochemical Quantum Yields Measured as Optical Density Steps}

To demonstrate the operating mode of the apparatus we describe as an example the photobleaching of thionine $\left(\mathrm{TH}^{+}\right)$by $\mathrm{p}$-iodoaniline in methanolic solution (sample II) since this reaction shows a typical magnetic field effect as will be shown below. Without considering mechanistic details, the net reaction can be described by the process:

$$
\mathrm{TH}^{+} \underset{\text { p-iodoaniline }}{\stackrel{h v}{\longrightarrow}} \mathrm{TH}_{2} \text {. }
$$

The reduced species $\mathrm{TH}_{2}$ (leucothionine) is colorless, the dye $\mathrm{TH}^{+}$ can be regenerated by re-oxidation with oxygen. The spectral change observed in the visible when illuminating the oxygen-free methanolic solution II with light of the $578 \mathrm{~nm}$ mercury line is shown in Fig. 2. The reaction has a fairly low quantum yield of $0.24 \%$ in the beginning and it decreases strongly in the course of the reaction. In view of the small quantum yield we consider this reaction a suitable case to demonstrate the efficiency of our method.

In Fig. 3 we show the time dependence of the optical density measured at $598 \mathrm{~nm}$ in the second flow-through cuvette when the solution is flowing and the first cuvette is irradiated by various light

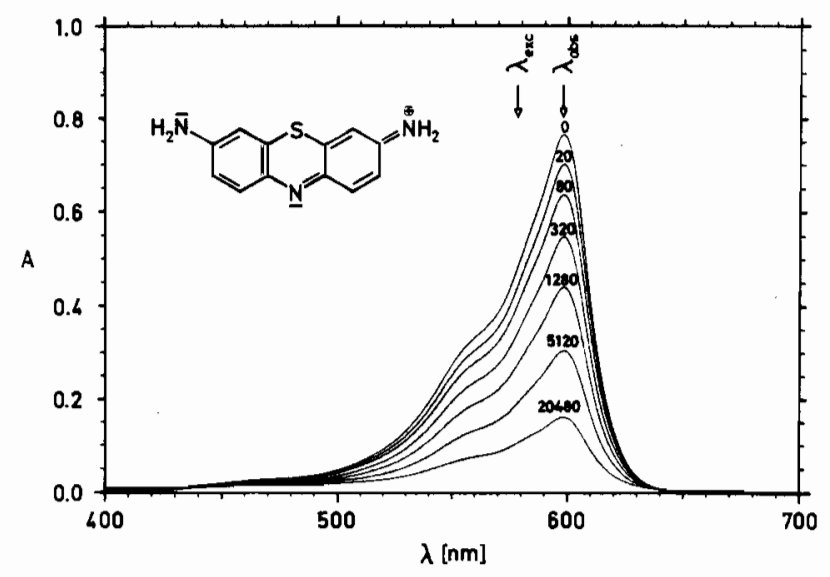

Fig. 2

Visible spectra of thionine in solution II after different periods of illumination. Illumination periods are given in seconds. The arrows indicate the wavelength of irradiation and the observation wavelength in the flow runs intensities. The photo diode signal monitoring the intensity of the actinic light is also shown in Fig. 3. It can be observed that a few seconds after the illumination is switched on, a sudden decrease of the optical density occurs in the spectrophotometer cuvette, the time lag being due to the transport time of the flow between the cuvettes. The signal levels off after a few more seconds, a time span which corresponds to the dwell time of the solution in the optical cavities of both cuvettes. As the light intensity is stepwise decreased, the optical density, too, changes in steps, now in the direction of increasing optical density, corresponding to decreased bleaching in the irradiation cuvette.

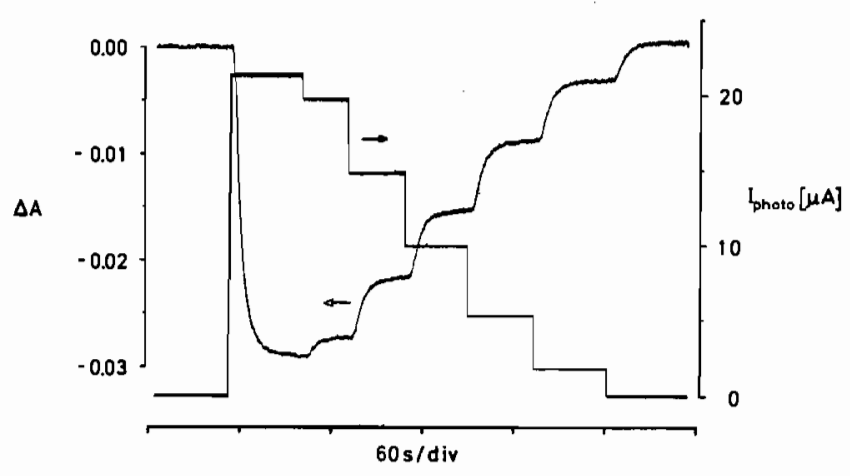

Fig. 3

YT-record of flow experiment with solution II (thionine/p-iodoaniline) under variation of illumination light flux.

a) Spectrophotometer response, left hand scale and

b) photocurrent indicating illumination light flux, right hand scale

As long as the total change of the optical density does not exceed $5 \%$ of the initial value, the correlation between illumination light intensity and the optical density recorded in the second cuvette is practically linear. Although this change of optical density corresponds to a fairly low degree of chemical turnover, it is generally sufficient to achieve a relative accuracy of $1 \%$ for most photoreactions if the noise level of the spectrophotometer is below $5 \cdot 10^{-4}$ optical density units.

It is obvious that changes in the illumination light intensity and changes in the quantum yield should produce equivalent optical density changes in this apparatus (cf. Eq. (1)). Thus the signal curve depicted in Fig. 3, where the optical density steps are produced by steps in the illumination light intensity, would be likewise obtained for constant illumination intensity if the photochemical quantum yield were magnetic field dependent and the magnetic field would be changed in a stepwise fashion. The linear correlation between optical density and illumination intensity should also hold true for the quantum yield.

\subsection{Magnetic Field Dependent Photochemical Quantum Yields}

Fig. 4 gives a demonstration of the magnetic field effect found for the permanent photochemical bleaching of thionine due to the reaction with p-iodoaniline in methanolic solution. As the magnetic field is switched between zero field and a certain value, corresponding steps of the optical density signal appear, indicating a decrease of the quantum yield with the magnetic field. The relative magnetic field effect $R(B)$ for a magnetic field $B$ is evaluated as the ratio of the $\Delta A$ step due to the magnetic field at full actinic light flux $(\Delta A(B))$ to the $\Delta A$ step due to the switching of the actinic light at zero field $\left(\Delta A_{\mathrm{ref}}\right)$. In Fig. 5 we show the magnetic field dependence of $R$.

If the reductive photobleaching of thionine in methanol is performed with aniline instead of $p$-iodoaniline no magnetic field effect is observed. However, if this reaction is performed in reversed micellar solution quite drastic magnetic field effects appear.

In Fig. 6 is shown the record of an illumination experiment with thionine and aniline in reversed micellar solution. As can be seen the magnetic field increases the permanent photobleaching quantum yield. Thus the sign of the magnetic field effect is opposite to 


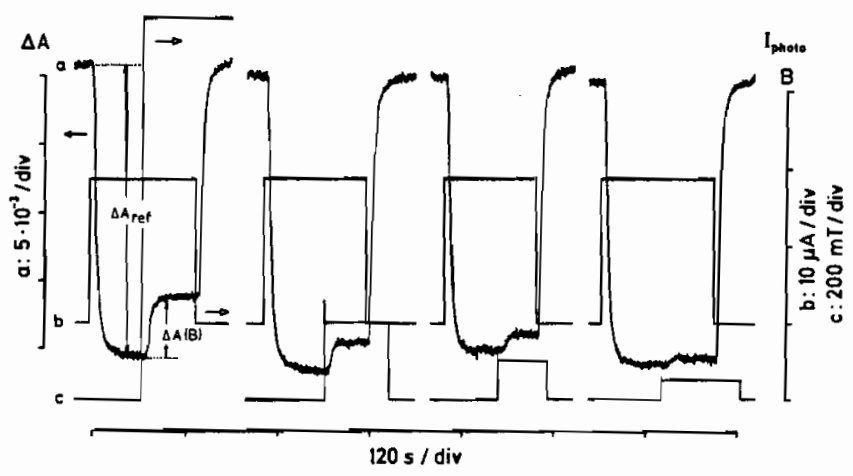

Fig. 4

YT-record of flow experiment with solution II (thionine/p-iodoaniline in methanol) under variation of the magnetic field.

a) Spectrophotometer response (left hand scale),

b) illumination light flux and

c) magnetic field strength ( $b$ and $c$ right hand scale, but with different zero levels)

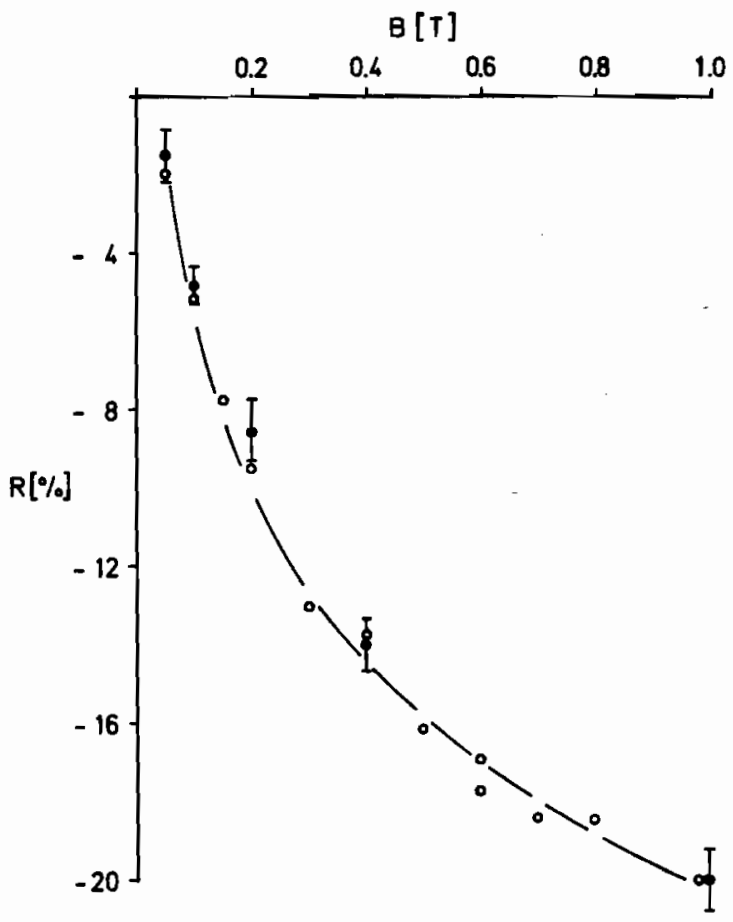

Fig. 5

Relative magnetic field effect $R(B)=\Delta A(B) / \Delta A_{\text {ref }}$ (cf. Fig. 4) on the photochemical quantum yields with solution II. Open circles: continuous illumination (determined from Fig. 4). Closed circles, previous results from laser flash experiments observing magnetic field effects on the radical yield [23]

the p-iodoaniline case. A diagram of the magnetic field effect $R(B)$ is shown in Fig. 7. We note that the typical field values necessary to observe sizeable effects are significantly lower than in the p-iodoaniline case (cf. Fig. 5).

\section{Discussion}

\subsection{Magnetic Field Effect According to the Radical Pair Mechanism}

During the last decade it has been well established by various methods as CIDNP, CIDEP and magnetic field effects in chemical kinetics (MARY [10]), that spin correlation effects in radical pairs (RPs) are essential for understanding
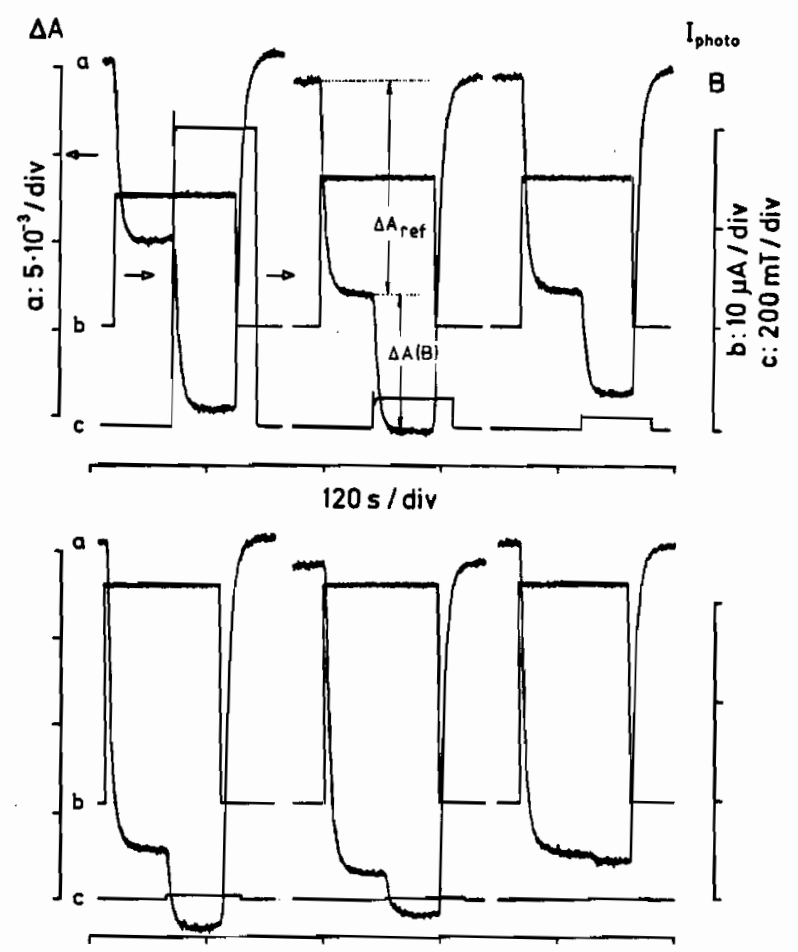

Fig. 6

YT-record of flow experiment with solution I (thionine with aniline in reversed micellar solution). For identification of signal traces cf. legend to Fig. 4

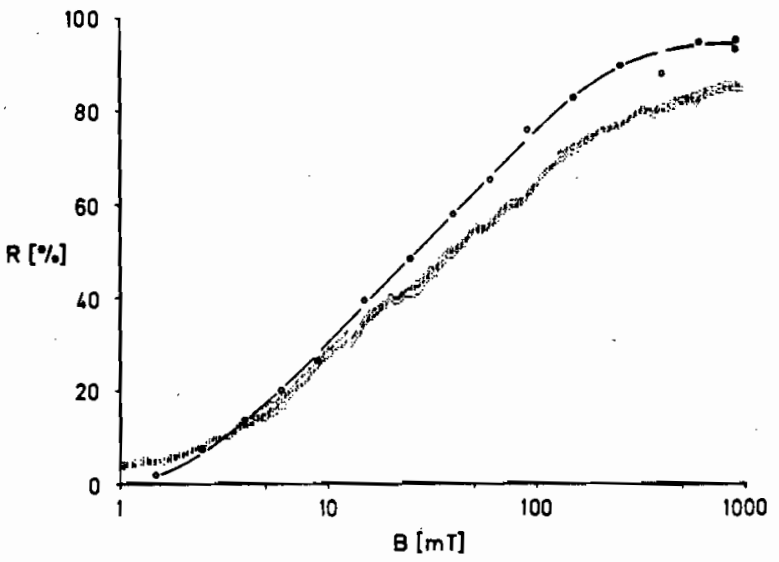

Fig. 7

Relative magnetic field effect $R(B)$ on the photobleaching quantum yield with solution I. Open circles: continuous illumination (determined from Fig. 6). Dotted trace: previous results from laser flash experiments in which magnetic field effects on the radical yield where observed [13]

their reactive behaviour (cf. Refs. $[1-3]$ ). The magnetic field effect on the spin motion in such RPs can serve as a handle to influence the distribution over the different reaction channels of the pair. It has been shown that the decay rate of the encounter probability of a geminate radical pair, which is closely related to the concept of the "cage effect", is a crucial parameter for the scale of the effects. Long cage times are favorable for the evolution of external magnetic field effects. From this point of view it is not surprising that in micelles, organized assemblies with the property of "super cages" $[11,12]$, particularly large magnetic field effects in RP reactions have been found. Recently we described a special example of such an effect which could be observed in 
the reaction of thionine triplet with aniline in the water core of reversed micelles $[13,14]$. The principle of this reaction and its magnetic field sensitivity is explained in Scheme I.

$$
\begin{aligned}
& \left({ }^{3} \mathrm{TH}^{+}\right)_{\text {mic }}+\mathrm{An} \stackrel{\mathrm{K}_{\mathrm{q}}}{\longrightarrow}{ }^{3}\left(\mathrm{TH} \cdot \mathrm{An}^{+} \cdot\right)_{\text {mic }} \\
& \left.\right|_{h v} \text {, isc } k_{31}||_{13} \stackrel{k_{\text {esc }}}{\longrightarrow} \mathrm{TH} \cdot+\left(A n^{+}\right)_{\text {mic }} \\
& \left(\mathrm{TH}^{+}\right)_{\text {mic }}+A n \underset{k_{\text {rec }}}{\leftarrow}\left(\mathrm{TH} \cdot A n^{+}\right)_{\text {mic }}
\end{aligned}
$$

Thionine $\left(\mathrm{TH}^{+}\right)$which may be solubilized in the water core of reversed micelles is optically excited and after intersystem crossing to its triplet state $\left({ }^{3} \mathrm{TH}^{+}\right)$abstracts an electron from aniline (An) to produce an intramicellar RP with initial triplet spin alignment. Intramicellar recombination can occur only via a RP triplet-singlet transition and competes with the escape of one of the radicals from the micelle into the unpolar bulk solvent. As our investigations have shown, in zero magnetic field the rate of diffusive encounters is rate determining for recombination rather than the establishment of RP spin equilibrium. By applying a magnetic field the triplet to singlet transitions of the RP become slower and hence more and more rate determining for the recombination reaction which is thus slowed down by the magnetic field. Since the escape rate is not magnetic field dependent, this leads to an increasing yield of free radicals when the field is increased. For a typical water pool radius of $1.5 \mathrm{~nm}$ the yield of escape radicals can be increased from $30 \%$ to almost $60 \%$ when increasing the magnetic field from zero field to 1 Tesla.

The free radicals decay in several milliseconds, however, the detailled kinetics of this decay has not been analyzed so far. In flash experiments the regeneration of thionine from the radicals seems almost complete, which does not exclude, however, a permanent bleaching yield of $0.47 \%$ as found in continuous illumination experiments at zero field. On the other hand, it is conceivable that the permanent bleaching reaction might not involve radical intermediates, if two redox equivalents are transferred in one step (cf. Ref. [15]). The search for a magnetic field effect on the permanent bleaching quantum yield appears as a conclusive experiment to decide this mechanistic question.

In Fig. 7 there are depicted four (almost identical) signal traces, adopted from our previous work [13] where the magnetic field effect on the escape yield of radicals was detected by means of microsecond laser flash photolysis at different delay times after the laser pulse. It is quite obvious that the laser experiments and the present continuous illumination experiments show very similar magnetic field effects which clearly documents that the radicals, produced in the triplet reaction and escaping from the micelles, are direct intermediates in the bleaching reaction. Furthermore, the similarity of the magnetic field effects on the free radicals yield and on the quantum yield of permanent bleaching indicates, that no sizable magnetic field effects occur in the free radical reactions leading to leucothionine formation.

From the zero field radical yield of $30 \%$ and the permanent bleaching yield of $0.47 \%$ it follows that the radical recombination leads to $98.4 \%$ thionine regeneration and to $1.6 \%$ leucothionine formation.
The following radical reactions have to be considered

$$
\begin{aligned}
& \mathrm{TH}^{\bullet}+\left(\mathrm{An}^{+}\right)_{\text {mic }} \rightarrow\left(\mathrm{TH}^{+}\right)_{\text {mic }}+\mathrm{An} \\
& 2 \mathrm{TH}^{\bullet}+\left(\mathrm{H}^{+}\right)_{\text {mic }} \rightarrow \mathrm{TH}_{2}+\left(\mathrm{TH}^{+}\right)_{\text {mic }} \\
& \mathrm{TH}_{2}+(\mathrm{An}+)_{\text {mic }} \rightarrow \mathrm{TH}^{\bullet}+\mathrm{An}+\left(\mathrm{H}^{+}\right)_{\text {mic }} \\
& \left(\mathrm{An}^{+}\right)_{\text {mic }}+\mathrm{An}+\mathrm{TH}^{\bullet} \rightarrow\left(\mathrm{An}_{2}^{+}\right)_{0 \mathrm{x}}+\mathrm{TH}_{2}
\end{aligned}
$$

i.e. recombination of thionine and aniline radicals (Eq. (3)), leading to direct thionine regeneration, the disproportionation of semithionine [16] (Eq. (4)) and, in view of the small quantum yield of leucothionine formation, we think it is necessary to assume the reoxidation of leucothionine by aniline radicals (Eq. (5)), since reaction (3) is not likely to be much faster than reaction (4). Of course, in order to retain some leucothionine, there must be also a reaction allowing for an independent disappearance of the aniline radicals (Eq. (6)). Since leucothionine is accumulated as the reaction proceeds, reaction (5) becomes more and more dominant over reaction (6), a fact which can explain the drastic decrease of the overall quantum yield in the course of the reaction (cf. Fig. 1).

With regard to the redox potentials available (all vs. s.c.e.):

$$
\begin{array}{lll}
E^{0}\left(\mathrm{TH}^{\cdot} / \mathrm{TH}^{+}\right)=-0.49 \mathrm{~V} & (\operatorname{Ref} .[17]) \\
E^{0}\left(\mathrm{An} / \mathrm{An}{ }^{+}\right)=0.87 \mathrm{~V} & (\operatorname{Ref} .[18]) \\
E^{0}\left(\mathrm{TH}_{2} / \mathrm{TH}_{2}^{+\cdot}\right)=0.06 \mathrm{~V} & (\operatorname{Ref.}[19])
\end{array}
$$

it is certainly reasonable to postulate reactions (3) and (5). The species $\left(\mathrm{An}_{2}^{+}\right)_{\mathrm{x} x}$ appearing in Eq. (6) might be identified with some benzidine or diphenylamine derivative as suggested by the results of electrochemical oxidation experiments with anilines $[20,21]$.

\subsection{Magnetic Field Effect According to the Triplet Mechanism}

Magnetic fields cannot only influence the reactive behaviour of RPs but also of excited triplet states, although the kinetic conditions to observe a photochemical quantum yield modulation according to the triplet mechanism are rather stringent and therefore examples of these effects are not as common as for the RP-mechanism. In fact, at present, the dissociation of heavy atom substituted triplet exciplexes as reported previously by Steiner et al. $[22,23]$ seem to be the only examples of magnetic field dependent chemical kinetics where the triplet mechanism has been invoked and applied for a detailled quantitative analysis of the magnetic field effect in liquid solution.

In these triplet exciplexes, which have to be assumed as intermediates in the electron transfer reaction between thionine triplet and halogen anilines [24, 25], intersystem crossing to the ground state competes with exciplex dissociation to the free radicals. This explains why the absolute radical yield decreases as the spin-orbit coupling constant of the heavy atom substituent increases. As could be rationalized, the heavy-atom-induced isc process in these exciplexes must be spin-sublevel selective, disfavouring isc from 
one of the triplet substates (viz $\mathrm{T}_{\mathrm{z}}$ ) which therefore is more effective in radical formation than the other two substates. The effect of an external magnetic field is to couple this $T_{z^{-}}$ substate to $T_{x}$ and $T_{y}$ which undergo fast isc. Therefore the external magnetic field contributes to increase the overall isc efficiency and to decrease the overall free radical yield.

In homogeneous solutions where these systems have been studied, the geminate RPs (here without Coulomb attraction) separate too fast as to contribute a significant magnetic field effect by the RP-mechanism, i.e. the magnetic field effect in these systems is almost entirely due to the triplet mechanism.

For the thionine/p-iodoaniline system the magnetic field dependence of the free radical yield as observed by laser flash spectroscopy is shown in Fig. 6. The coincidence with the magnetic field effect on the permanent photobleaching yield as observed in this work is almost perfect.

From the flash experiments we know that in zero field the radical formation efficiency amounts to $13.5 \%$. As in the thionine/aniline case, described above, thionine regeneration from the radicals is almost complete, except for a weak permanent reductive bleaching as described in section 3.1. with a quantum yield of $0.24 \%$ (it should be noted that the quantum yield ratio of free radical production to final leucothionine formation is of the same order of magnitude as in the thionine/aniline system).

From the great similarity of the magnetic field effects on radical yield and permanent bleaching yield it has to be concluded that the magnetic field effect on the free radical yield, which was explicable in terms of the heavy-atom-enhanced triplet mechanism, is directly transferred to the permanent bleaching yield. Again, this strongly indicates

a) that leucothionine formation starts from thionine triplet and occurs via two one-electron transfer steps as described in Eqs. $(3-6)$ and

b) that the free radical reactions themselves are not noticeably modified by a magnetic field.

\section{Conclusion}

In this paper we have demonstrated that by a relatively simple technique the magnetic field dependence of photochemical quantum yields can be measured with high precision. In our experiments the accuracy was limited by a $\Delta A$ noise of about $5 \cdot 10^{-4}$ optical density units (cf. Figs. 3, 4, 6 ), which, however, by using a longer time constant of the detection system (several seconds) should be reducible to about $2 \cdot 10^{-4}$ optical density units. Thus, with the experimental set up described, absolute quantum yield changes of $10^{-4}$ and relative quantum yield changes of $10^{-2}$ are detectable. In cases where the reference $\Delta A$-step might be increased up to 0.3 (in our experiments it was in the order of 0.03 ), a relative accuracy of $10^{-3}$ seems within reach. Thus very small magnetic field effects should become detectable and investigations of this kind might establish themselves as a valuable routine method for obtaining mechanistic information on photochemical reactions. Detecting the reaction yield by absorption spectroscopy will be feasible for most photoreactions, but for special cases the potentiality of the technique could be extended by using fluorescence spectroscopy instead. Especially laser-induced fluorescence can be made a very sensitive detection method for photochemical products.

Finally one should be aware of the fact that not only static magnetic fields but also microwave irradiation can modulate photochemical reaction yields [26]. The experimental technique described in this paper appears also promising for performing reaction yield detected magnetic resonance (RYDMR) experiments which should give even more detailled information in that the electron spin resonance spectra of specific reaction intermediates can be obtained.

Financial support of this investigation by the Deutsche Forschungsgemeinschaft and the Fonds der Chemischen Industrie is gratefully acknowledged.

\section{References}

[1] K. M. Salikhov, Yu. N. Molin, R. Z. Sagdeev, and A. L. Buchachenko, "Spin Polarization and Magnetic Effects in Radical Reactions", Vol. 22, in: "Studies in physical and theoretical chemistry", Yu. N. Molin, ed., Elsevier, Amsterdam 1984.

[2] N. J. Turro, Proc. Nat1. Acad. Sci. USA 80, 603 (1983).

[3] K. Schulten and A. Weller, Biophys. J. 24, 295 (1978); A. Weller. H. Staerk, and R. Treichel, Faraday Discuss. Chem. Soc. 78, 271 (1984).

[4] "Chemically Induced Magnetic Polarization", Proc. of the NATO Adv. Study Institute at Sogesto, Urbino, Italy. Eds. L. T. Muus, P. W. Atkins. K. A. McLauchlan, and J. B. Pedersen, Reidel, Dordrecht. Holland 1977.

[5] F. J. Adrian, Rev. Chem. Intermed. 3, 3 (1979).

[6] J. K. S. Wan, Adv. Photochem. 12, 283 (1980).

[7] H. Mauser, „Formale Kinetik“, Bertelsmann Universitätsverlag, Düsseldorf 1974.

[8] R. G. Lawler and M. Halfon, Rev. Sci. Instrum. 45, 84 (1974).

[9] W. Endriss, PhD Thesis, Universität Stuttgart 1961

[10] W. Lersch and M. E. Michel-Beyerle, Chem. Phys. 78, 115 (1983).

[11] N. J. Turro, Pure Appl. Chem. 53, 259 (1981).

[12] N. J. Turro and G. C. Weed, J. Am. Chem. Soc. 105, 1861 (1983).

[13] W. Schlenker, T. Ulrich, and U. E. Steiner, Chem. Phys. Lett. 103, $118(1983)$

[14] T. Ulrich and U. E. Steiner. Chem. Phys. Lett. 112, 365 (1984).

[15] P. Hemmerich, W.-R. Knappe, H. E. A. Kramer, and R. Traber, Eur. J. Biochem. 104, 511 (1980).

[16] a) H. Fischer, Z. physik. Chem. Neue Folge 43, 177 (1964); b) R. Bonneau, J. Faure, and J. Joussot-Dubien, Ber. Bunsenges. Phys, Chem. 72, 263 (1968); c) H. Fischer, H. E. A. Kramer, and A. Maute, Z. physik. Chem. N. F. 69, 113 (1970).

[17] E. Vogelmann, S. Schreiner, W. Rauscher, and H. E. A. Kramer, Z. physik. Chem. Neue Folge 101, 321 (1976).

[18] K. Sasaki, A. Kitani, and M. Tsuboi, Nippon Kagaku Kaishi 12. 2269 (1973).

[19] Calculated from two-electron redox potential and semiquinone formation constant (cf. Ref. [17]).

[20] J. Bacon and R. N. Adams, J. Am. Chem. Soc. 90, 6596 (1968).

[21] R. L. Hand and R. F. Nelson, J. Am. Chem. Soc. 96, 850 (1974).

[22] U. Steiner, Ber. Bunsenges. Phys. Chem. 85, 228 (1981).

[23] T. Ulrich. U. E. Steiner, and R. E. Föll, J. Phys. Chem. 87, 1873 (1983).

[24] U. Steiner and G. Winter, Chem. Phys. Lett. 55, 364 (1978).

[25] G. Winter and U. Steiner. Ber. Bunsenges. Phys. Chem. 84, $1203(1980)$.

[26] E. L. Frankevich and S. I. Kubarev, in: "Triplet State ODMR Spectroscopy", p. 137, ed. R. H. Clarke, Wiley Interscience, New York 1982. 